\title{
Electron-phonon interactions and two-phonon modes associated with charge density wave in single crystalline $1 \mathrm{~T}-\mathrm{VSe}_{2}$
}

\author{
Juhi Pandey (ib and Ajay Soni ${ }^{*}{ }^{*}$ \\ School of Basic Sciences, Indian Institute of Technology Mandi, Mandi 175075, Himachal Pradesh, India
}

(Received 23 March 2020; revised 21 April 2020; accepted 28 June 2020; published 22 July 2020)

\begin{abstract}
The charge density wave (CDW) is a unique phenomenon mostly realized in two-dimensional (2D) metallic layered transition metal dichalcogenides. We report on the observation of commensurate CDW (C-CDW) and incommensurate (I-CDW) transition in single crystal $1 \mathrm{~T}-\mathrm{VSe}_{2}$ using the spectroscopy technique in the temperature range of 50-120 K. The room temperature Raman spectra showed a sharp $A_{1 g}$ mode $\sim 206 \mathrm{~cm}^{-1}$ along with two additional modes associated to $E_{g} \sim 257 \mathrm{~cm}^{-1}$ symmetry and two-phonon $\left(2_{p h}\right) \sim 332 \mathrm{~cm}^{-1}$ interactions. The onset temperature of I-CDW and C-CDW is estimated from resistance measurements supported by magnetic measurements. Remarkably, at the onset of I-CDW $\sim 115 \mathrm{~K}$, a significant enhancement in the intensity of the weak $E_{g}$ mode is observed along with emergence of a doubly degenerate $E_{g}(2)$ mode $\sim 144 \mathrm{~cm}^{-1}$, while below $70 \mathrm{~K}$, the emergence of the $A_{1 g}$ mode $\sim 170 \mathrm{~cm}^{-1}$ signifies the onset of C-CDW. The observation of a sudden rise in the intensity of the $E_{g}$ mode $\sim 257 \mathrm{~cm}^{-1}$ mode, below I-CDW, showed the involvement of electron-phonon coupling in $1 \mathrm{~T}-\mathrm{VSe}_{2}$.
\end{abstract}

DOI: 10.1103/PhysRevResearch.2.033118

\section{INTRODUCTION}

The charge density wave (CDW) is a low-temperature ordered phase that arises from periodic modulation of conduction electron densities accompanied by periodic distortion of the atomic lattice [1-3]. The commonly accepted argument for the origin of the CDW, in many low-dimensional systems, is based on the Peierls picture described for purely onedimensional metallic systems, where electronic instabilities result in Fermi-surface nesting. Here, the CDW is said to be driven by Fermi-surface nesting since the contour of a Fermi surface coincides with ordering vector $\boldsymbol{Q}$ [4]. However, there are several recent reports suggesting that nesting alone is insufficient to explain the CDW. In many cases, especially for two dimensions (2D) and higher dimensions, the Fermi surface does not nest at the ordering vector $\boldsymbol{Q}[5,6]$. For instance, in the cases of $2 \mathrm{H}-\mathrm{NbSe}_{2}, \mathrm{CeTe}_{3}$, and $\mathrm{ErTe}_{3}$, strong electron-phonon coupling plays a more important role for determining $\boldsymbol{Q}$ than Fermi-surface nesting [7,8]. Based on the periodicity of $\boldsymbol{Q}$, th eCDW has been classified as commensurate CDW (C-CDW), nearly commensurate (N-CDW), and incommensurate CDW (I-CDW) with a high-symmetry phase. In the C-CDW phase, the $\boldsymbol{Q}$ is an integral multiple of the reciprocal lattice vector of the undistorted phase, whereas in the I-CDW phase it is not [9].

\footnotetext{
*ajay@iitmandi.ac.in

Published by the American Physical Society under the terms of the Creative Commons Attribution 4.0 International license. Further distribution of this work must maintain attribution to the author(s) and the published article's title, journal citation, and DOI.
}

Layered transition metal dichalcogenides (TMDCs) provide a rich platform for research with their wide range of physical properties varying from metallic, semiconducting, superconducting, and magnetism to having exotic optical properties [10-12]. Most 2D materials exist in two stable crystal structures such as $2 \mathrm{H}$ (trigonal prismatic) and $1 \mathrm{~T}$ (octahedron), where the physical properties depend on the symmetry of the phases [13]. Among the several versatile properties, the CDW is a novel phenomenon realized in $1 \mathrm{~T}$ and $2 \mathrm{H}$ polytypes of many metallic layered TMDCs, such as $\mathrm{NbSe}_{2}, \mathrm{TaSe}_{2}, \mathrm{TaS}_{2}, \mathrm{VTe}_{2}$, and $\mathrm{VSe}_{2}$ [14-20]. Thus, these materials have received much scientific and technological attention for many-body interactions and potential applications in memory devices, transistors, logic-gate electronics, and oscillators [14-20]. The origin of the CDW in TMDCs has not achieved any general scientific consensus and has remained a topic of investigation so far [5,21-24]. For 2D systems such as $2 \mathrm{H}-\mathrm{NbSe}_{2}, 2 \mathrm{H}-\mathrm{TaS}_{2}$, and $2 \mathrm{H}-\mathrm{TaSe}_{2}$ [25,26], electronphonon coupling plays a major role in CDW compared to Fermi-surface nesting. 1T-VSe 2 is isostructural with $1 \mathrm{~T}-\mathrm{TaS}_{2}$ and $1 \mathrm{~T}-\mathrm{TaSe}_{2},[9,24,27]$ and is expected to show similar anomaly in resistivity measurement at the CDW, but unlike these isostructural compounds showing a sharp anomaly in resistivity at $\mathrm{CDW}$ transitions [28,29], $\mathrm{VSe}_{2}$ shows a gradual upturn similar to $2 \mathrm{H}-\mathrm{NbSe}_{2}, 2 \mathrm{H}-\mathrm{TaS}_{2}$, and $2 \mathrm{H}-\mathrm{TaSe}_{2}$ [30-32].

$\mathrm{VSe}_{2}$ is a compound that has been intensively studied for its interesting electronic band structure and CDW. The bulk $\mathrm{VSe}_{2}$ crystallizes in the $1 \mathrm{~T}$ polytype, where the $\mathrm{V}$ atoms are covalently bonded with the octahedra of Se atoms to form a single layer of $\mathrm{VSe}_{2}$ and these layers are further stacked through van der Waals interactions [33]. 1T-VSe 2 shows I-CDW transition $\sim 110 \mathrm{~K}$ and $\mathrm{C}$-CDW transition $\sim 80 \mathrm{~K}$ which have been studied using the X-ray diffraction technique, [9] inelastic $x$-ray scattering technique, angle-resolved 
photoemission spectroscopy [34], and transport measurement [35]. However, because of the high reflectivity of this compound, the obtained optical signals are extremely poor; thus several properties such as vibrational properties, and electronphonon coupling are not well understood. Thus, in this paper, we have studied the electron-phonon coupling driven CDW transitions in bulk single crystal $1 \mathrm{~T}-\mathrm{VSe}_{2}$, using temperaturedependent resistivity, magnetic susceptibility, and Raman spectroscopy measurements. The coupling between electron and phonon, at low temperatures, is demonstrated from resistivity as well as magnetic measurements, while spectroscopy is used as a tool to access the CDW in $\mathrm{VSe}_{2}$.

\section{EXPERIMENTAL SECTION}

A single crystal of $\mathrm{VSe}_{2}$ was grown by reacting a stoichiometric amount of vanadium chips and selenium pellets (both from Sigma-Aldrich, 99.9\%) using the chemical vapor transport method with iodine as the transport agent $\left(\sim 2 \mathrm{~kg} / \mathrm{m}^{3}\right)$. The reactants were vacuum sealed in a quartz ampoule and placed in a two-zone furnace, which was heated slowly $(\sim 20 \mathrm{~h})$ to reach the growth temperatures of $1093 \mathrm{~K}$ (hot zone) and $923 \mathrm{~K}$ (cold zone). The samples were kept in the gradient temperature for $\sim 72 \mathrm{~h}$ followed by slow cooling to room temperature over $\sim 20 \mathrm{~h}$. Thus, large shiny crystals of $\mathrm{VSe}_{2}$ were obtained at the cold end of the tube. X-ray diffraction (XRD) of the prepared crystals was obtained using a rotating anode Rigaku SmartLab diffractometer in BraggBrentano geometry with $\mathrm{Cu} K \alpha$ radiation $(\lambda=1.5406 \AA)$. To determine the crystal structure and phase purity, Rietveld refinement of the XRD patterns was done using FULLPROF SUITE software [36].

Four-probe electrical resistance $(R)$ and magnetic susceptibility $(\chi)$ measurements were performed from 2 to $300 \mathrm{~K}$ using a Quantum Design physical properties measurement system and magnetic properties measurement system, respectively. Micro-Raman measurements were done using a Horiba Jobin-Yvon LabRAM HR evolution Raman spectrometer in backscattering geometry with solid state laser excitation $(\sim 532 \mathrm{~nm}), 1800$ grooves $/ \mathrm{mm}$ grating, and a Peltier cooled charge-doupled device (CCD) detector. Polarized Raman measurements were performed using two halfwave plates as polarizer and analyzer. Temperature-dependent micro-Raman measurement was performed by using a Montana cryostation in the temperature range of 3-300 K. All the Raman measurements are performed on the (001) plane of $1 \mathrm{~T}-\mathrm{VSe}_{2}$ single crystal. Field emission scanning electron microscopy (FESEM) images and elemental mapping using energy dispersive spectroscopy (EDS) were recorded through JFEI Nova Nano SEM-450 (see Supplemental Material [37]).

\section{RESULTS AND DISCUSSION}

The XRD pattern and Rietveld refinement of the $\mathrm{VSe}_{2}$ is shown in Fig. 1(a). The estimated lattice parameters are $a=3.361 \AA$ and $c=6.101 \AA$, with a unit cell volume $=59.726 \AA^{3}$. The difference curve $\left(Y_{\text {obs }}-Y_{\text {calc }}\right)$ clearly shows the grown $\mathrm{VSe}_{2}$ crystals are of highquality single phase while the single crystalline nature is confirmed by observation of peaks only along the $c$

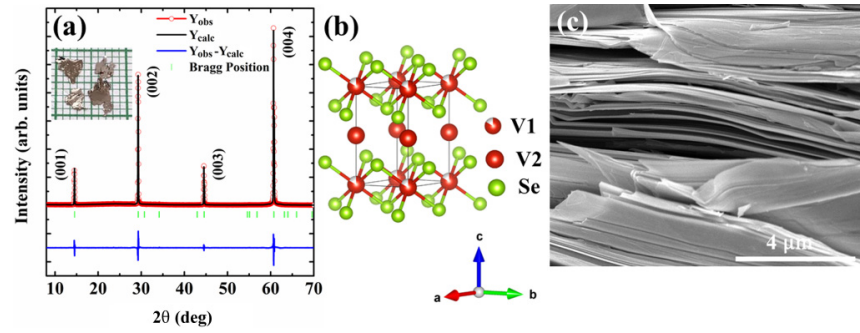

FIG. 1. (a) XRD pattern of single crystal of $1 \mathrm{~T}-\mathrm{VSe}_{2}$; inset shows the optical image of the grown crystals. (b) Schematic of trigonal crystal structure of $P \overline{3} m 1$ space group. (c) FESEM imaging of the layered nature of the crystal.

axis. The inset shows the optical image of the grown crystals with large dimensions $(\sim 5 \mathrm{~mm} \times 5 \mathrm{~mm})$. In general, the $\mathrm{VSe}_{2}$ has trigonal crystal structure with a $P \overline{3} m 1$ space group [Fig. 1(b)]; however, there is a mild possibility of self-intercalation of $\mathrm{V}$ atoms between the van der Waals gap of $\mathrm{VSe}_{2}$, thus occupying two different sites, V1 $(0,0,0)$ and V2 $(0,0,0.5)$ [38]. Such self-intercalation is commonly observed due to synthesis at high temperature [39]. Nevertheless, the extent of self-intercalation can be minimized by synthesizing the compound at a lower temperature, $\sim 923 \mathrm{~K}$. The layered nature of the $\mathrm{VSe}_{2}$ crystal is observed through FESEM imaging shown in Fig. 1(c). The stoichiometry of the prepared sample has been estimated using elemental mapping (see Supplemental Material, Table S1 [37]).

The temperature-dependent electrical resistance $(R)$ shows metallic behavior throughout the $T$ range with an onset of the hump below $110 \mathrm{~K}$ [Fig. 2(a)]. In general, the close association of structural changes and modifications of the charge density are represented by a hump in $R(T)$, which is a signature of CDW transition in most of the materials. The observations are in line with several layered TMDCs such as $1 \mathrm{~T}-\mathrm{TaS}_{2}$, $1 \mathrm{~T}-\mathrm{TaSe}_{2}$, and $2 \mathrm{H}-\mathrm{TaSe}_{2}$ [28]. Additionally, the quality of the crystal plays an important role for the onset of CDW transition, and the residual resistance ratio, defined as RRR = $R_{300 \mathrm{~K}} / R_{6.2 \mathrm{~K}}$, is one of the parameters to estimate. Here, the RRR is about 8 , which is in good agreement with previous reports and confirms the high quality of the synthesized $\mathrm{VSe}_{2}$ single crystal [35]. We have assessed the temperature range of the CDW transition from temperature derivative of resistivity, $d R / d T$, where two points of inflection at $T \sim 102 \mathrm{~K}$ and $\sim 50 \mathrm{~K}$ have been observed. In support to our observations, the temperature-dependent $\mathrm{x}$-ray diffractions studies by Tsutsumi [9] described the periodic lattice distortion wave vector in the reciprocal lattice as $(0.250 \pm 0.003) a^{*}+(0.314 \pm 0.003) c^{*}$ in the I-CDW state below $110 \mathrm{~K}$ and $(0.250 \pm 0.003) a^{*}+$ $(0.307 \pm 0.003) c^{*}$ in the C-CDW state below $85 \mathrm{~K}$. Here, the $a^{*}$ component is independent of temperature; however, the $c^{*}$ component shows deviation with temperature suggesting that the origin of incommensurability is along the $c$ axis. Thus, the observed inflections in $d R / d T$ are associated to I-CDW $\sim$ $102 \mathrm{~K}$ and $\mathrm{C}-\mathrm{CDW} \sim 50 \mathrm{~K}$.

The inset in Fig. 2(a) shows a low-temperature region below $10 \mathrm{~K}$, where $R$ decreases with temperature and exhibits a minimum at $\sim 6.2 \mathrm{~K}$ and then increases up to $2 \mathrm{~K}$. This lowtemperature upturn in $R(T)$ can originate from various reasons 


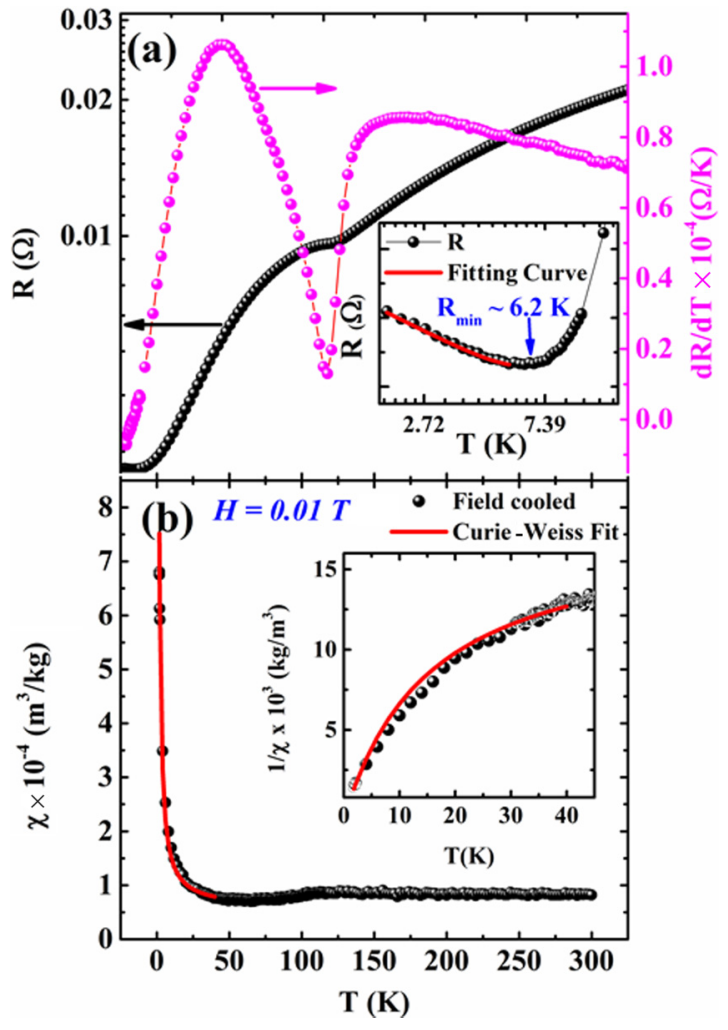

FIG. 2. (a) Resistance $(R)$ and first derivative of resistance $(d R / d T)$ of $1 \mathrm{~T}-\mathrm{VSe}_{2}$; inset shows upturn in $R$ below $6.2 \mathrm{~K}$ due to Kondo interactions. (b) Variation of magnetic susceptibility $(\chi)$ with temperature showing downturn at the onset of CDW. The inset figure shows the variation of $1 / \chi$ with temperature fitted with modified Curie-Weiss law.

such as weak localization, electron-electron interactions, and the Kondo effect [40,41]. Recently, Barua et al. [42] observed a similar upturn in resistivity $(\rho)$ of $1 \mathrm{~T}-\mathrm{VSe}_{2}$ appearing due to $\mathrm{V}$ ions intercalated between the van der Waals gap, which contributed to magnetic susceptibility and acted as localized magnetic scatterers to the conduction electrons. Here, in the case of $\mathrm{VSe}_{2}$, the intercalated $\mathrm{V}^{4+}$ ions with an unpaired electron in the $d$ orbital can provide magnetic moments which can drive the electron transport at low temperatures $[39,43,44]$. This kind of localized magnetic impurity can result in scattering of conduction electrons through $s-d$ exchange interactions and the observed upturn in $R$ (or $\rho$ ) through such interactions is described as the Kondo effect [42]. We have fitted the lowtemperature $\rho$ with $\rho=\rho_{0}+\alpha T^{2}+c_{m} \ln \left(\frac{T_{K}}{T}\right)+b T^{5}$, where $\rho_{0}$ is the residual resistivity, $\alpha T^{2}$ shows the contribution from the Fermi liquid properties, the term $b T^{5}$ is from the lattice vibrations, and $c_{m}\left(\ln \frac{T_{K}}{T}\right)$ represents the contribution from the Kondo effect. Below $6.2 \mathrm{~K}$ the logarithmic Kondo term is dominating over other negligible parameters except $\rho_{0}$. Thus fitting of the upturn of $R$ below $10 \mathrm{~K}$ is suggesting the existence of Kondo-like behavior in $\mathrm{VSe}_{2}$.

To confirm the presence of magnetic moments in $1 \mathrm{~T}-\mathrm{VSe}_{2}$ because of the intercalated $\mathrm{V}^{4+}$ and its effect on the CDW, the temperature-dependent magnetic susceptibility $(\chi)$ has been performed and the data are shown in Fig. 2(b). The $\chi$ shows a downturn at the onset of the $\mathrm{CDW}$ in $R$ measurement. The in-

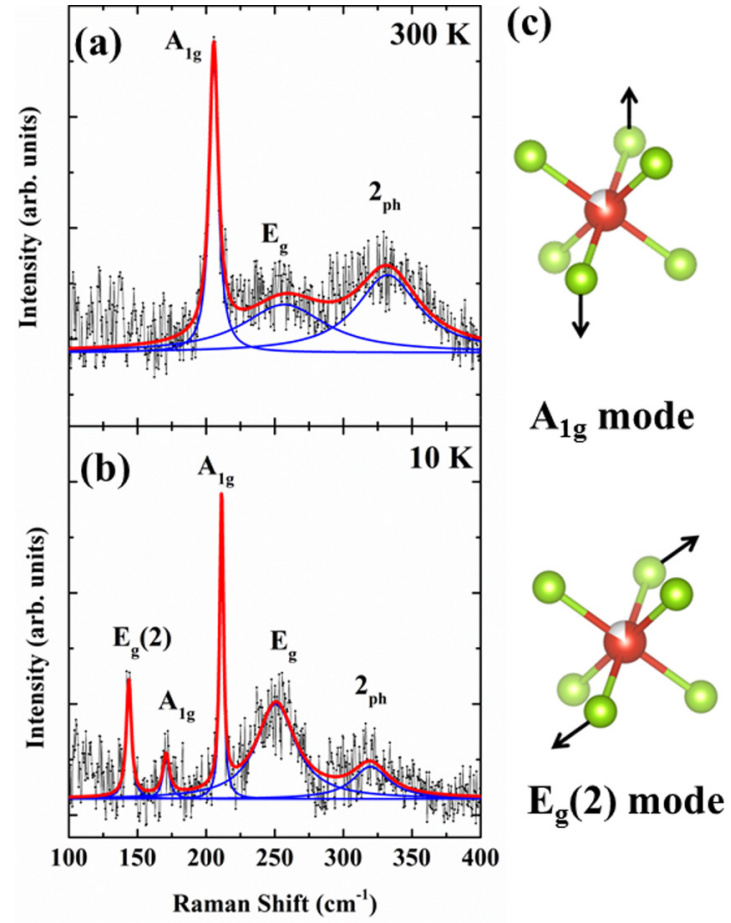

FIG. 3. Raman spectra of $1 \mathrm{~T}-\mathrm{VSe}_{2}$ at (a) $300 \mathrm{~K}$ and (b) $10 \mathrm{~K}$; (c) schematic of main type of lattice vibrations observed in Raman spectra.

set in Fig. 2(b) shows the $1 / \chi$ from 1.8 to $40 \mathrm{~K}$ (field $\sim 0.01 \mathrm{~T}$ ) fitted with a modified Curie-Weiss law $\chi(T)=\frac{C}{T-\theta}+\chi_{0}$, where $\chi_{0}$ is the temperature-independent intrinsic contribution to susceptibility from the $\mathrm{V}$ bands, $C$ is a Curie constant, and $\theta$ is a paramagnetic Curie temperature. The parameters obtained from $1 / \chi$ fitting using the modified Curie-Weiss law are $\chi_{0}=0.00005 \mathrm{~m}^{3} / \mathrm{kg}, C=0.0009 \mathrm{~m}^{3} / \mathrm{kg} \mathrm{K}$, and $\theta=$ $0.5 \mathrm{~K}$. Thus, the intercalated $\mathrm{V}$ is contributing to the feeble magnetism in the sample (shown in Fig. S1, in the Supplemental Material [37]). After confirming the quality of the sample and observation of the signature CDW from low-temperature measurements, we have performed the Raman spectroscopy to understand the phenomena in detail [24].

At high temperatures above the CDW transition, the formula unit of $\mathrm{VSe}_{2}$ has three atoms per unit cell [30,45], and thus has nine zone-center vibrational modes, which can be presented with irreducible representation, $\Gamma=A_{1 g}+E_{g}(2)+$ $2 A_{2 u}+2 E_{u}(2)$. Here only two modes, $A_{1 g}$ and the doubly degenerate $E_{g}(2)$ modes, are Raman active while the other four are IR active modes. [13] Figure 3(a) shows Raman spectra at $300 \mathrm{~K}$ having three peaks at $\sim 206, \sim 257$, and $\sim 332 \mathrm{~cm}^{-1}$. For $\mathrm{VSe}_{2}$, Sugai et al. [46] reported that the sharp peak at $\sim 206 \mathrm{~cm}^{-1}$ is $A_{1 g}$ symmetry. Due to the lack of group theoretical analysis of vibrational modes for $1 \mathrm{~T}-\mathrm{VSe}_{2}$, the origin of the two peaks, $\sim 257 \mathrm{~cm}^{-1}$ and $\sim 332 \mathrm{~cm}^{-1}$, could not be understood and thus requires further investigations. To confirm the origin of these modes, we have performed the polarized Raman spectra of the $\mathrm{VSe}_{2}$ crystal (Fig. S2, Supplemental Material [37]), where the laser is incident normal to the $a b$ plane. For parallel polarization $(X X)$ configuration, the incident and the scattered light are polarized along the same 
axis, while for perpendicular polarization $(X Y)$ configuration, they are orthogonal. The sharp $A_{1 g}$ mode is observed only in parallel polarization while the mode $\sim 257 \mathrm{~cm}^{-1}$ is present in both parallel as well as perpendicular polarization measurements [46]. Thus, the mode at $\sim 257 \mathrm{~cm}^{-1}$ has $E_{g}$ symmetry. Further, considering the fact that $1 \mathrm{~T}-\mathrm{TiSe}_{2}$ has similar crystal structure and comparable molecular masses of $\mathrm{V}$ and $\mathrm{Ti}$, the origin of these modes can be consigned using the knowledge of the vibrational modes of $1 \mathrm{~T}-\mathrm{TiSe}_{2}[45,47]$. Similar to the broad modes, $\sim 257 \mathrm{~cm}^{-1}$ and $332 \mathrm{~cm}^{-1}$, in $1 \mathrm{~T}-\mathrm{VSS}_{2}$, weak and broad features are observed at $\sim 300 \mathrm{~cm}^{-1}$ and $360 \mathrm{~cm}^{-1}$ for $1 \mathrm{~T}-\mathrm{TiSe}_{2}$ which are assigned to $A_{1 g}$ symmetry and the two-phonon process $[30,47]$. Therefore, the broad feature $\sim 332 \mathrm{~cm}^{-1}$ for $1 \mathrm{~T}-\mathrm{VSe}_{2}$ can be related with the two-phonon process $(2 p h)[30]$.

The low-temperature (10 K) Raman spectra [Fig. 3(b)] has five Raman modes and is quite distinct from the $300 \mathrm{~K}$ spectra. The peaks observed, $\sim 144 \mathrm{~cm}^{-1}, \sim 171 \mathrm{~cm}^{-1}, \sim 211 \mathrm{~cm}^{-1}$, $\sim 251 \mathrm{~cm}^{-1}$, and $320 \mathrm{~cm}^{-1}$, are assigned to doubly degenerate the $E_{g}(2), A_{1 g}, A_{1 g}, E_{g}$, and $2_{p h}$ modes, respectively [48]. The symmetry assigned for all the modes is based on the symmetry operation on an undisturbed $1 \mathrm{~T}-\mathrm{VSe}_{2}$ crystal. The schematic of the vibrational modes of $A_{1 g} \sim 206 \mathrm{~cm}^{-1}$ and $E_{g}(2)$ are shown in Fig. 3(c). Here, the $A_{1 g}$ mode represents out-of-plane vibration (along the $c$ axis) of Se atoms, while the doubly degenerate $E_{g}(2)$ mode is associated with the opposite vibration of Se atoms relative to each other along the $a$ axis and $b$ axis. The $E_{g}(2)$ mode is expected at $300 \mathrm{~K}$ as per the irreducible representation; however, owing to the weak scattering, the mode remains hidden in the noise at $300 \mathrm{~K}$ and the intensity of this mode enhances below $120 \mathrm{~K}$. The Raman spectrum shown in Fig. 3 is base line corrected and normalized with the intensity of the $A_{1 g}$ mode, $\sim 206 \mathrm{~cm}^{-1}$.

To understand the role of the CDW on phonons, we performed a temperature-dependent Raman study from 300 to $10 \mathrm{~K}$, as shown in Fig. 4 (also in Supplemental Material [37], Fig. S3). From 300 to $120 \mathrm{~K}$, three Raman modes, $A_{1 g}$, $E_{g} E_{g}$, and $2_{p h}$, have been observed; however, the spectral features are broad and weakly intense for $E_{g}$ and $2 p h$. The resemblance in the Raman spectrum from 300 to $120 \mathrm{~K}$ confirms the stability of the trigonal structure up to $120 \mathrm{~K}$. However, below $120 \mathrm{~K}$, the intensity of the broad $E_{g}$ mode starts to rise significantly and becomes comparable with the intensity of the most intense $A_{1 g}$ mode. Further, the doubly degenerate $E_{g}(2)$ mode starts to appear prominently between 100 and $10 \mathrm{~K}$ (shown in the red rectangle). Such modulation in the Raman spectra with temperature is related with the onset of the CDW transition below $120 \mathrm{~K}$, as the size of the unit cell increases due to the formation of superlattices [46]. Thus, the vibrational spectra are dramatically modulated by the formation of superlattices, which have been probed by Raman and Fourier transform infrared (FTIR) studies [49].

In the case of incommensurate distortions, the translational symmetry of the lattice is lost and the restriction of first-order Raman scattering to zone-center phonons is relaxed, resulting in several additional modes in the first-order Raman scattering. Interestingly, a very minor peak of $A_{1 g} \sim 171 \mathrm{~cm}^{-1}$ appears at the $40 \mathrm{~K}$ spectra and grows further in low-temperature spectra (shown in green rectangle), as reflected in $R$ measurement for the onset of the commensurate phase below $\sim 50 \mathrm{~K}$.

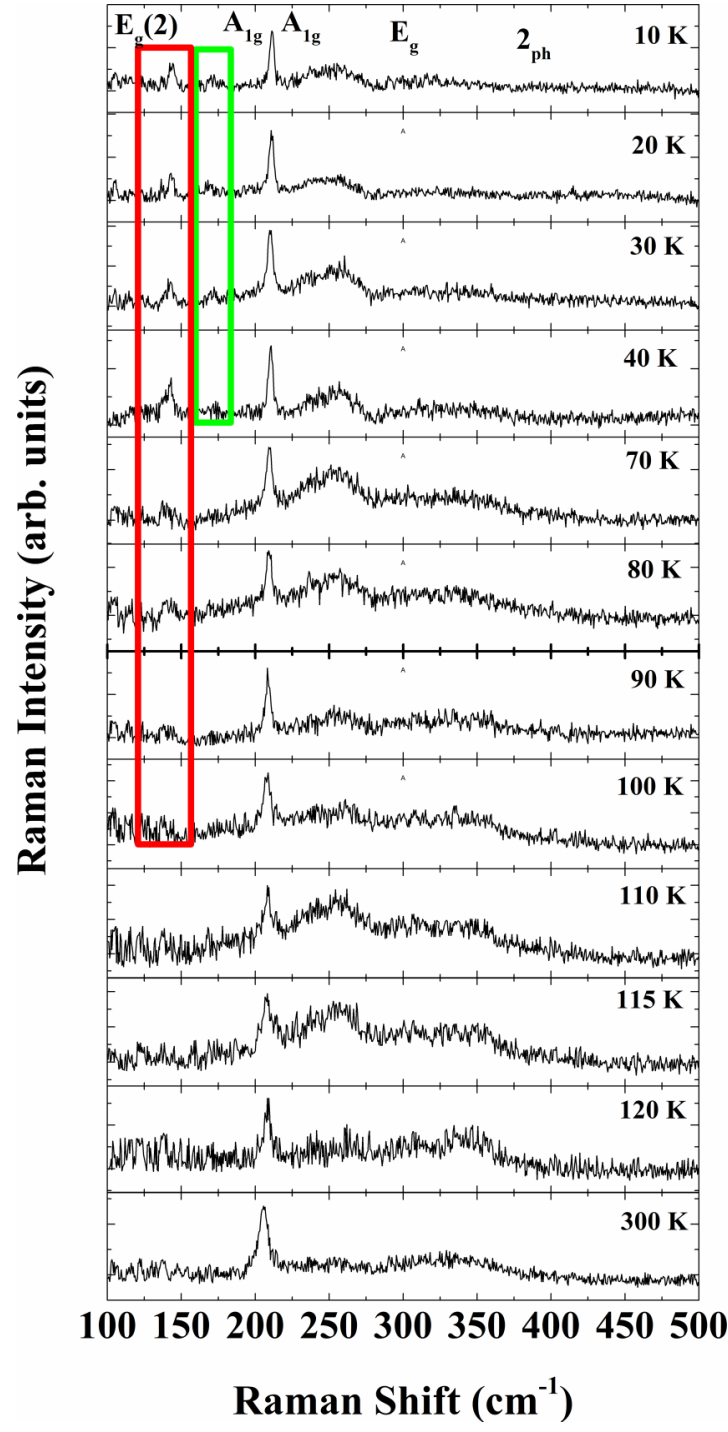

FIG. 4. Temperature dependence of Raman spectra from 300 to $10 \mathrm{~K}$. The enclosed red and green rectangles represent the emergence of $E_{g}(2)$ at the I-CDW and $A_{g}$ modes at the C-CDW, respectively.

The behavior can be understood by the formation of a commensurate superlattice where the size of the Brillouin zone reduces, which results in the folding of phonon dispersion curves [24]. This folding can lead to the appearance of new zone-center phonons compared to the undistorted structure. Therefore, the appearance of the out-of-plane $A_{1 g}$ mode below $50 \mathrm{~K}$ can be associated with the formation of a commensurate superlattice. Further, earlier studies on x-ray investigation of a superlattice at I-CDW and C-CDW transitions demonstrated the significant variation along the $c^{*}$ axis at the C-CDW transition, whereas the component along the $a^{*}$ axis remains independent of temperature; therefore the appearance of the out-of-plane $A_{1 g}$ mode at $\sim 50 \mathrm{~K}$ is a signature of C-CDW transition.

To explore the correlation between the CDW transition and phonon energy, the variation of the peak position of the $E_{g}(2)$ mode, the $A_{1 g}$ mode $\sim 211 \mathrm{~cm}^{-1}$, and the $E_{g}$ mode are shown in Fig. 5(a), and the integrated intensity of the $E_{g}$ mode is shown in Fig. 5(b). The peak position and the integrated 

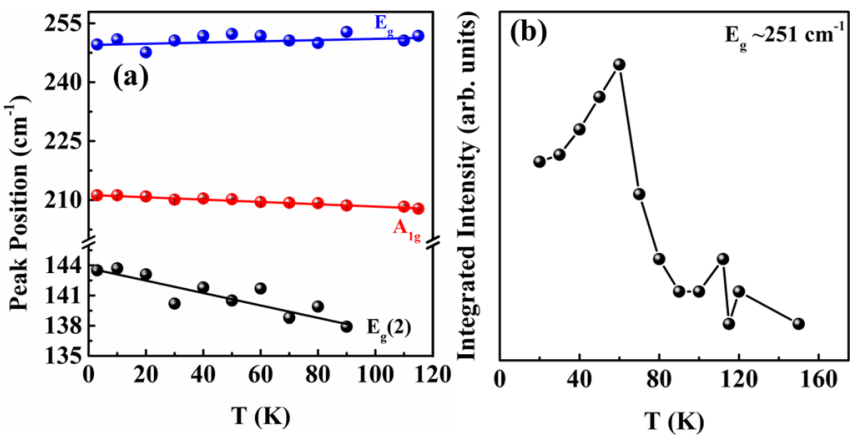

FIG. 5. Temperature dependence of (a) the peak position of the vibrational modes with temperature, and (b) integrated intensity of $E_{g}$ mode as observed in the normalized Raman spectra.

intensity are determined through the Lorentzian peak fitting of each normalized Raman spectrum. The $E_{g}(2)$ mode and the $A_{g}$ mode $\sim 211 \mathrm{~cm}^{-1}$ show phonon hardening on the lowering of temperature up to $10 \mathrm{~K}$. These modes have been fitted with $\omega(T)=\omega_{0}+m T$, where $\omega_{0}$ is the phonon frequency of the $E_{g}(2)$ and $A_{g}$ modes at absolute zero temperature; $m$ is the first-order temperature coefficient of the two modes [50]. The fitted curve is shown as a solid line in Fig. 5(a) and the $m$ values for the $E_{g}(2)$ and $A_{g}$ modes obtained by the linear fitting are -0.0617 and $-0.0296 \mathrm{~cm}^{-1} / \mathrm{K}$, respectively. The stiffening of the phonon mode estimated from $m$ depends upon anharmonic coupling of phonons and thermal expansion of the crystal. In contrast to these two modes showing the usual phonon hardening, the broad $E_{g}$ mode does not show a significant change in energy with temperature as shown with the solid blue line in Fig. 5(a). However, the integrated intensity of the $E_{g}$ mode which is active in both the CDW transitions enhances strongly below the I-CDW transition temperature $\sim 100 \mathrm{~K}$ [Fig. 5(b)]. Further, it is worth noticing that the integrated intensity reaches maxima near C-CDW transition and then starts to decline at further lower temperature suggesting the coupling of Raman modes with CDW transitions. After the CDW transition, the density of electronic states coupled with phonons decreases at the Fermi surface, which results in the decrease of Raman intensity at further lower temperature. Further, the decrease of the Raman intensity below the C-CDW suggests the deformation of the crystal structure [51]. Thus, the emergence of new Raman modes represents the formation of a superlattice at the onset of the CDW transition and strong coupling of the $E_{g}$ mode with the CDW, giving evidence of electron-phonon coupling.

\section{CONCLUSION}

In summary, the novel phenomenon of the CDW has been investigated in single crystalline $1 \mathrm{~T}-\mathrm{VSe}_{2}$ using temperaturedependent resistance measurement and Raman spectroscopy. The hump in resistance measurement below $110 \mathrm{~K}$ is related to the onset of the I-CDW, while the existence of the C$\mathrm{CDW} \sim 50 \mathrm{~K}$ is confirmed by $d R / d T$. The appearance of five Raman active modes at low temperature suggested the reduction in the size of the Brillouin zone due to the formation of superlattices. The onset of the $\mathrm{C}-\mathrm{CDW}$ transition is marked with the appearance of the $A_{1 g}$ mode $\sim 177 \mathrm{~cm}^{-1}$. At the onset of the I-CDW, the intensity of the $E_{g}$ mode, $\sim 257 \mathrm{~cm}^{-1}$, enhanced significantly and the $E_{g}(2)$ mode $\sim 114 \mathrm{~cm}^{-1}$ becomes prominent. The rise of the integrated intensity of the $E_{g}$ mode at the onset of the CDW transitions signifies the role of electron-phonon coupling in the CDW transition.

\section{ACKNOWLEDGMENTS}

We would like to thank IIT Mandi for the instruments and research facilities. A.S. would like to acknowledge DSTSERB for funding (Grant No. CRG/2018/002197). J.P. acknowledges MHRD, India for providing a fellowship for the research work.
[1] G. Grüner, Rev. Mod. Phys. 60, 1129 (1988).

[2] P. Leininger, D. Chernyshov, A. Bosak, H. Berger, and D. S. Inosov, Phys. Rev. B 83, 233101 (2011).

[3] D. S. Inosov, V. B. Zabolotnyy, D. V. Evtushinsky, A. A. Kordyuk, B. Büchner, R. Follath, H. Berger, and S. V. Borisenko, New J. Phys. 10, 125027 (2008).

[4] W. Kohn, Phys. Rev. Lett. 2, 393 (1959).

[5] C. M. Varma and A. L. Simons, Phys. Rev. Lett. 51, 138 (1983).

[6] M. Lavagnini, M. Baldini, A. Sacchetti, D. DiCastro, B. Delley, R. Monnier, J. H. Chu, N. Ru, I. R. Fisher, P. Postorino, and L. Degiorgi, Phys. Rev. B 78, 201101(R)(2008).

[7] H.-M. Eiter, M. Lavagninia, R. Hackl, E. A. Nowadnick, A. F. Kemper, T. P. Devereaux, J.-H. Chu, J. G. Analytis, I. R. Fisher, and L. Degiorgi, Proc. Natl. Acad. Sci. USA 110, 64 (2013).

[8] F. Weber, S. Rosenkranz, J.-P. Castellan, R. Osborn, R. Hott, R. Heid, K.-P. Bohnen, T. Egami, A. H. Said, and D. Reznik, Phys. Rev. Lett. 107, 107403 (2011).

[9] K. Tsutsumi, Phys. Rev. B 26, 5756 (1982).

[10] J. A. Wilson and A. D. Yoffe, Adv. Phys. 18, 193 (1969).
[11] A. V. Stier, N. P. Wilson, K. A. Velizhanin, J. Kono, X. Xu, and S. A. Crooker, Phys. Rev. Lett. 120, 057405 (2018).

[12] J. Pandey and A. Soni, Appl. Surf. Sci. 463, 52 (2019).

[13] J. Ribeiro-Soares, R. M. Almeida, E. B. Barros, P. T. Araujo, M. S. Dresselhaus, L. G. Cançado, and A. Jorio, Phys. Rev. B 90, 115438 (2014).

[14] G. Liu, B. Debnath, T. R. Pope, T. T. Salguero, R. K. Lake, and A. A. Balandin, Nat. Nanotechnol. 11, 845 (2016).

[15] G. Liu, S. Rumyantsev, M. A. Bloodgood, T. T. Salguero, and A. A. Balandin, Nano Lett. 18, 3630 (2018).

[16] M. Yoshida, R. Suzuki, Y. Zhang, M. Nakano, and Y. Iwasa, Sci. Adv. 1, e1500606 (2015).

[17] G. Liu, E. X. Zhang, C. D. Liang, M. A. Bloodgood, T. T. Salguero, D. M. Fleetwood, and A. A. Balandin, IEEE Electron Device Lett. 38, 1724 (2017).

[18] A. K. Geremew et al., Nanoscale 11, 8380 (2019).

[19] A. G. Khitun, A. K. Geremew, and A. A. Balandin, IEEE Electron Device Lett. 39, 1449 (2018).

[20] A. K. Geremew et al., ACS Nano 13, 7231 (2019). 
[21] K. Rossnagel, J. Phys.: Condens. Matter 23, 213001 (2011).

[22] W. L. McMillan, Phys. Rev. B 16, 643 (1977).

[23] M. D. Johannes and I. I. Mazin, Phys. Rev. B 77, 165135 (2008).

[24] R. Samnakay, D. Wickramaratne, T. R. Pope, R. K. Lake, T. T. Salguero, and A. A. Balandin, Nano Lett. 15, 2965 (2015).

[25] J. Zhu, H. Li, L. Zhong, P. Xiao, X. Xu, X. Yang, Z. Zhao, and J. Li, ACS Catal. 4, 2917 (2014).

[26] P. Hajiyev, C. Cong, C. Qiu, and T. Yu, Sci. Rep. 3, 2593 (2013).

[27] S. Tanda, T. Sambongi, T. Tani, and S. Tanaka, J. Phys. Soc. Jpn. 53, 476 (1984).

[28] F. J. Di Salvo and J. E. Graebner, Solid State Commun. 23, 825 (1977).

[29] S. Sun, L. Wei, Z. Li, G. Cao, Y. Liu, W. J. Lu, Y. P. Sun, H. Tian, H. Yang, and J. Li, Phys. Rev. B 92, 224303 (2015).

[30] P. Goli, J. Khan, D. Wickramaratne, R. K. Lake, and A. A. Balandin, Nano Lett. 12, 5941 (2012).

[31] P. Garoche, J. J. Veyssié, P. Manuel, and P. Molinié, Solid State Commun. 19, 455 (1976).

[32] T. Valla, A. V. Fedorov, P. D. Johnson, P. A. Glans, C. McGuinness, K. E. Smith, E. Y. Andrei, and H. Berger, Phys. Rev. Lett. 92, 086401 (2004).

[33] M. Hossain, Z. Zhao, W. Wen, X. Wang, J. Wu, and L. Xie, Crystals 7, 298 (2017).

[34] K. Terashima, T. Sato, H. Komatsu, T. Takahashi, N. Maeda, and K. Hayashi, Phys. Rev. B 68, 155108 (2003).

[35] C. Yadav and A. Rastogi, Solid State Commun. 150, 648 (2010).
[36] R. A. Young, The Rietveld Method (Crystallography/Oxford University Press, Oxford, 1993).

[37] See Supplemental Material at http://link.aps.org/supplemental/ 10.1103/PhysRevResearch.2.033118 for Elemental mapping, magnetic hysteresis and Raman measurements.

[38] J. Rigoult, C. Guidi-Morosini, A. Tomas, and P. Molinie, Acta Crystallogr., Sect. B 38, 1557 (1982).

[39] F. J. DiSalvo and J. V. Waszczak, Phys. Rev. B 23, 457 (1981).

[40] S. Zhang et al., Adv. Mater. 21, 2282 (2009).

[41] W. Niu, M. Gao, X. Wang, F. Song, J. Du, X. Wang, Y. Xu, and R. Zhang, Sci. Rep. 6, 26081 (2016).

[42] S. Barua, M. Hatnean, M. Lees, and G. Balakrishnan, Sci. Rep. 7, 10964 (2017).

[43] M. Bayard and M. J. Sienko, J. Solid State Chem. 19, 325 (1976).

[44] S. Acharya, S. Anwar, T. Mori, and A. Soni, J. Mater. Chem. C 6, 6489 (2018).

[45] Y. Takaoka and K. Motizuki, J. Phys. Soc. Jpn. 49, 1838 (1980).

[46] S. Sugai, K. Murase, S. Uchida, and S. Tanaka, J. Phys. Colloq. 42, C6-740 (1981).

[47] J. A. Holy, K. C. Woo, M. V. Klein, and F. C. Brown, Phys. Rev. B 16, 3628 (1977).

[48] J. E. Smith, J. C. Tsang, and M. W. Shafer, Solid State Commun. 19, 283 (1976).

[49] N. Nagaosa and E. Hanamura, Phys. Rev. B 29, 2060 (1984).

[50] I. Calizo, A. A. Balandin, W. Bao, F. Miao, and C. N. Lau, Nano Lett. 7, 2645 (2007).

[51] Y. Hu, F. Zheng, X. Ren, J. Feng, and Y. Li, Phys. Rev. B 91, 144502 (2015). 